\section{Japanese psychiatry}

SIR-As the other visiting scientist named in the article by Alun Anderson entitled "Abuse for visiting scientists" (Nature 315,$361 ; 1985$ ), I would like to add to Kimio Moriyama's response (Nature 318, $308 ; 1985)$. Dr Moriyama writes that his group did not "intend to visit the smaller meeting at Nagoya". At the smaller meeting, Dr Crow and I were the only two speakers to be scheduled and our presentations were cancelled because the group of psychiatrists and their followers from Tokyo and Gifu were on their way to disrupt the meeting. Previous encounters had led to violent fights between the two groups. I fully agree with $\mathrm{Dr}$ Crow (Nature319, 172; 1986). I too am willing to have the abstracts of the cancelled meeting of the Japanese Biological Psychiatric Society, deemed by Dr Moriyama to be unethical, examined by an official Institutional Review Board. I hope that the Japanese psychiatric patients, abandoned by their families, will not be deprived of participation in psychiatric research.

There are apparently some changes now to the betterment of patient rights and patient care in Japan. Changes like that are always too slow. Violent threats and intimidation belong to another era. I do not believe that anyone is served when psychiatrists go into fist fights to disrupt meetings. It will do nothing to remove the stigma attached to the mentally ill or to increase public support for reform. Presumably, we are all involved, because we want to improve our patients' lot and believe in the principle of freedom of scientific information.

Daniel P. van Kammen Veterans Administration Medical Center, Highland Drive,

Pittsburgh, Pennsylvania 15206, USA

\section{Wasting assets}

SIR-I recently attended a one-day international meeting concerned with a fundamental aspect of physics. For twentyfour hours I was free to do my proper job of thinking about science and how mankind can better understand the sensible universe. Such is the contrast between this pleasant and productive interlude and what my life otherwise has become that I actually felt guilty at absenting myself from the usual Sisyphean labour of trying to overcome inadequacy of resources, digging away at piles of unproductive paperwork, making meaningless returns and providing unvalidated "indicators" of performance. It was like attending a party in the penthouse of a building while the foundations are crumbling.

A number of overseas colleagues expressed disquiet at the crisis in funding of British science and education, and even more at the crisis in morale resulting from continual insults and deprecation, and our despair that the nature and importance of what we do will ever be understood. It became apparent that we have come to mistrust our politicians far more than do scientists of other countries. As a transatlantic colleague put it, while US politicians do indeed devote much attention to the need to be re-elected every few years, they are sophisticated enough to understand that pure knowledge and research are the seed-corn of what the culture and industry of their country will need in ten to thirty years' time.

By contrast, we encounter pennypinching which is squandering a great national asset, once esteemed as among the finest in the world. The whole structure of our institutions concerned with knowledge, from schools through universities to original research, is in disarray. One can hardly doubt that if present policies continue, then even apart from explicit support for research, we shall lack the structures that enable talented people to be educated and become the creative researchers of the future.

In the absence of the new ideas generated by research, we shall lack an essential ingredient needed for industrial prosperity in future decades. This situation has within it the seeds of Britain becoming a third world country, taking its ideas and industrial capital from more developed countries.

The question is not whether we can afford what we spend on schools, universities and research, but how a densely populated island can afford to spend so little on cultivating its intellectual assets. The knowledge base of our culture and industry is in jeopardy so long as we invest less on it than we wager on horses.

Peter Fellgett

University of Reading,

Department of Cybernetics,

3 Earley Gate, Whiteknights,

Reading RG6 $2 A L, U K$

\section{Creationism}

SIR-We have witnessed, again, the basic underlying view of creationism in D.H. Koobs' letter (Nature 319, 172; 1986), where he shows four of the methods creationists commonly use during their assaults on science.

First it is claimed that science does not have a complete understanding (which it never can) of important items such as the origin of life or the Universe, that these are some sort of boundaries to reality, that they are impermeable to human scrutiny and that since they are boundaries not crossable by science they are therefore, by default, proof of their presupposed supernatural creator. Nothing is potentially beyond science.

Secondly, Koobs brings science down to the creationists' level by making the absurd statement that there are areas of science that are to be taken on faith. I have heard many creationists claim it is a greater leap of faith to believe in evolution than it is to believe in creation. These people use that word incorrectly. Faith is a belief without evidence, and in the case of creationism it is a belief in spite of evidence. However, we all know that science accepts nothing on faith.

Thirdly, Koobs gives the impression that reality is manifested by human emotions and desires with this statement: "... who are free to choose which history provides more meaning for life". No comment is necessary.

Finally, misrepresentation runs rampant in creationism. Koobs is obviously referring to punctuated equilibrium when he claims that animals preserved in the fossil record "occurred spontaneously" and trying to equate punctuated equilibrium and spontaneous generation as acts of creation.

Creationism and science will never be two different ways of looking at reality. Creationists do their best to twist, lie, fabricate, misrepresent all they can of reality to deceive their followers and the lay public.

J. RiCHARD WAKEFIELD

385 Main Street,

Beaverton, Ontario, Canada LOK 1 AO

\section{Animal deception?}

SiR-Several times recently I have read claims (most recently in Nature 319, 143145 ; 1986) that animals practise deception. There appears to be a strange logic at work here.

First, we have a Cartesian animal, with a limited repertoire of behaviours. There is one behaviour called "alarm call", which the investigator has presumably defined very carefully by extensive observation.

Next, it is reported that the "alarm call" has been used for some other purpose. Instead of concluding that his initial definition of "alarm call" was too narrow, the investigator summons up another animal, one which uses the "alarm call" deceptively. This second, non-Cartesian animal is thus implicitly given credit, or blame, for an act of choice, which invokes a presumption of self-consciousness, even an ethical being.

Ruppell (the author's first citation) may have started this fad with his account of the "lying" mother fox. I believe the investigator is deceiving the investigator here. At the very least, words like "deception", with strong ethical connotations, seem out of place in this sort of context.

Research Foundation of

Herbert McArthur

State University of New York, State University Plaza,

Albany, New York 12246, USA 